\title{
The role of Carbon Capture and Storage electricity in attaining 1.5 and $2^{\circ} \mathrm{C}$
}

\author{
Adriano Vinca*2, Marianna Rottoli ${ }^{4}$, Giacomo Marangoni ${ }^{1,5}$ and Massimo \\ Tavoni ${ }^{3,5}$ \\ ${ }^{1}$ Earth and Environmental Systems Institute, Penn State University, University Park, PA, USA \\ ${ }^{2}$ International Institute for Applied Systems Analysis (IIASA), Laxenburg, Austria \\ ${ }^{3}$ Politecnico di Milano, Milan, Italy \\ ${ }^{4}$ Potsdam Institute for Climate Impact Research (PIK), Potsdam, Germany \\ ${ }^{5}$ RFF-CMCC European Institute on Economics and the Environment, Milan, Italy
}

\begin{abstract}
The climate targets defined under the Paris agreement of limiting global temperature increase below 1.5 or $2^{\circ} \mathrm{C}$ require massive deployment of low-carbon options in the energy mix, which is currently dominated by fossil fuels. Scenarios suggest that Carbon Capture and Storage (CCS) might play a central role in this transformation, but CCS deployment is stagnating and doubts remain about its techno-economic feasibility. In this article, we carry out a throughout assessment of the role of CCS electricity for a variety of temperature targets, from 1.5 to above $4^{\circ} \mathrm{C}$, with particular attention to the lower end of this range. We collect the latest data on CCS economic and technological future prospects to accurately represent several types of CCS plants in the WITCH energy-economy model, We capture uncertainties by means of extensive sensitivity analysis in parameters regarding plants technical aspects, as well as costs and technological progress. Our research suggests that stringent temperature scenarios constrain fossil fuel CCS based deployment, which is maximum for medium policy targets. On the other hand, Biomass CCS, along with renewables, increases with the temperature stringency. Moreover, the relative importance of cost and performance parameters change with the climate target. Cost uncertainty matters in less stringent policy cases, whereas performance matters for lower temperature targets.
\end{abstract}

*Corresponding author: vinca@iiasa.ac.at

IIASA, Schlossplatz 1 - A-2361 Laxenburg, Austria 
Keywords: Carbon Capture and Storage, Integrated Assessment Model, Climate mitigation policies, Electricity sector, Low-carbon technology, 1.5 and $2^{\circ} \mathrm{C}$ targets

\section{Introduction}

Following a continuous increase in scientific awareness of anthropogenic climate change, in 2015 the Paris agreement opened a call for policies more ambitious than ever before, aimed at limiting global temperature increase well below $2^{\circ} \mathrm{C}$, and in the direction of $1.5^{\circ} \mathrm{C}$ (IPCC, 2014). The objective of achieving such stringent targets entails a shift towards low carbon energy technologies. This implies a non-trivial transformation of the status quo, where fossil fuels account for more than $80 \%$ of the global primary energy supply (IEA, 2016) due to their dispatchability, economic convenience and technological maturity.

Given that the dependence on fossil fuels is not likely to decline in the short term (IEA, 2013a), many sources agree that CCS, among the possible low-carbon options available for energy production, might provide an important contribution to the sector decarbonization (IPCC, 2014; IEA, 2013a; OECD/IEA, 2010). Some multi model comparisons show that CCS might represent from $30 \%$ to $40 \%$ of the primary energy use (Koelbl et al., 2014), and biomass is likely to be the most used fuel combined with CCS, especially at the end of the century (Van Der Zwaan et al., 2013). Krey et al. (2014), showed that CCS and biomass constrains are those mostly influencing the results of the $2^{\circ} \mathrm{C}$ climate mitigation scenario. Little is however known about CCS potential in below 2 degrees scenarios, and different outcomes in models results seems to be dependent on CCS-related assumptions. The authors themselves suggest that further research would be advisable in order to provide a more detailed intra-model sensitivity analysis. Within this work we aim at contributing in addressing these literature gaps, providing a single model CCS centered analysis.

CCS potential lies mainly in the power sector, fossil fuels processing and industrial applications(Leung et al., 2014): a careful representation of demand structure all these sectors is therefore arguably recommended in a modeling exercise to provide meaningful results (Leeson et al., 2017; Muratori et al., 2017). Given the model architecture used in this analysis, which has a highly aggregated industrial sector, the authors preferred concentrating exclusively on the power sector, but stress the fact that a generic CCS based energy source for industry is included in the model.

CCS consists of separating and capturing fuel $\mathrm{CO}_{2}$ content at energy production and industrial facilities. CCS can be coupled with both fossil fuels and biomass (Bio CCS): if coupled with biomass, a negative emission level can be achieved (Kemper, 2015; IEAGHG,

2014a; Chum et al., 2011). Zero or negative emissions technologies are supposed to be 
crucial in limiting the temperature increase to $1.5^{\circ} \mathrm{C}$ in the next decades (Rogelj et al., 2015). Captured $\mathrm{CO}_{2}$ is later transported towards storage sites for long term confinement. CCS technologies offer some major advantages. First of all, they can significantly decrease emission levels without the need of shifting away from the currently used carbon-rich fuels. Secondly, power production is provided on-demand, being CCS a dispatchable technology. Furthermore, literature suggests that CCS availability in the technology portfolio could be associated with remarkable cost savings, for a given mitigation target (Davidson et al., 2017).

Despite these advantages, safety concerns about storage sites, public acceptance and high technology costs (see for example (Karayannis et al., 2014)) are often mentioned as major drawbacks, along with the lack of appropriate business models. Literature further claims that CCS large scale deployment is hardly achievable in the current policy scenario, which does not provide a stable and targeted support (Lipponen et al., 2017). Uncertainty concerning socioeconomic impact of large scale CCS deployment complicate the issue even more (Muratori et al., 2016; Creutzig et al., 2013).

Indeed, so far, CCS adoption is still limited to small scale plants, especially in the industrial sector, and its potential is subjected to large uncertainties (GCCSI, 2016).

It is therefore not yet clear to what extent CCS can effectively contribute to climate change mitigation effort (Koelbl et al., 2013, 2014; Metz et al., 2005), and in particular if large scale applications will make inroads in the electric sector (Whittaker and Kneppers, 2013). Moreover, particularly stringent climate targets such as the $1.5^{\circ} \mathrm{C}$ would increase competition among CCS technologies: this aspect could hinder fossil fuel based CCS deployment, encouraging a shift towards carbon neutral or carbon negative options (Bauer et al., 2015; Van der Zwaan and Tavoni, 2011; De Cian and Tavoni, 2012). This paper aims at contributing to the debate on the potential impact of CCS adoption on both the energy and the economic sector, offering a quantitative assessment of optimal CCS mitigation strategies in different future scenarios, with an emphasis on the role of $\mathrm{CO}_{2}$ capture rather than underground storage.

In particular, we tackle the following questions:

1. what is the state of the art of CCS technological options in the power sector?

2. to what extent could uncertainty of future cost and performances estimates impact CCS adoption?

3. how sensitive is CCS deployment to the climate policy target?

The first question is more qualitative and is best answered by reviewing the relevant literature. The data collected in the process was then implemented in the WITCH energy climate model to answer the remaining questions. Previous analyses have compared CCS 
potential in the $2^{\circ} \mathrm{C}$ scenario resulting from different Integrated Assessment Models, including WITCH (Luderer et al., 2012). This latter model resulted to have quite simplified and conservative assumptions concerning CCS, which have been expanded and updated for the purpose of this study. Moreover, the analysis presents extensive sensitivity analysis over input techno-economic parameters, as well as climate policy objectives, providing an exhaustive evaluation of CCS uncertainties for low end climate targets,

The paper is structured as follows. In Section 2, we present an overview of the CCS state of the art in the power sector. We also describe the WITCH model and the main changes included for this study. In Section 3 we present the results we obtained both from the literature review and the model output, providing answers to the research questions. In Section 4 we draw the conclusions.

\section{Materials and Methods}

\section{$2.1 \quad$ WITCH model}

WITCH is a dynamic global model, developed at Fondazione Eni Enrico Mattei (FEEM) and Centro Euro-Mediterraneo sui Cambiamenti Climatici (CMCC) and extensively used for cost-effective analyses of mitigation portfolios (Emmerling et al., 2016). The model fully integrates a top-down description of the economy sector with a bottom-up representation of the energy sector. Projections range from 2005 (the base year) up to 2150, with time steps of 5 years. The world economy is divided into 13 regions, where countries are clustered according to economic, energy and geographical similarities. Each region acts as a forward-looking planner, who optimizes the allocation of investments in final goods, innovation (R\&D) and energy technologies, with the objective to maximize social welfare. The overall solution is obtained via an open loop non-cooperative game among the regions. The game is solved through iterations as some decisions spill over on the decisions of other players. For example, investments in R\&D of one party not only improves the technologies in its energy system, but also those in other regions according to a given knowledge diffusion dynamics. The resulting investment paths define energy portfolios and associated costs, as well as the consequent greenhouse gas emission profiles into the climate system. GHG emissions affect global temperature, and in case of climate policies, are considered as environmental externalities.

The energy sector is designed through a nested CES production function, whose elasticities of substitution represent the observed heterogeneity in the energy system. Economic growth is driven by a mix of electric and non-electric energy services, which are supplied by a variety of options. The non-electric sector is aggregated by fuel type, while the electricity sector is 
more detailed and model specific power technologies. Among them, WITCH includes different coal based CCS alternatives, a generic natural gas technology and a generic biomass fueled plant with CCS. Each option group together power plants of similar type, which are then defined by a common set of key features, namely investment and operation and maintenance costs, plant net electricity efficiency (on LHV basis), carbon capture rate ratio (or capture efficiency), lifetime and capacity factor (annual operative hours). CCS technologies compete with all the other power technologies, spanning from fossil fuels -oil, coal and gas- plants, to nuclear and renewable technologies, such as hydropower, solar and wind. Technologies are subjected also to future progress, either via learning by doing or learning by researching. The former results in a cost reduction of CCS, wind and solar plants, as function of the installed capacity. The latter involves specific investments to reduce the cost of breakthrough advanced biofuels, advanced nuclear plants and vehicles batteries, or increase the efficiency of the energy system as a whole.

Land-use is modelled in WITCH by the mean response functions produced by the Global Biosphere Management Model (GLOBIOM) land-use model (Havlík et al., 2014). The GLOBIOM model provides biomass supply curves to the WITCH for each period and shared socio-economic scenario. These information define maximum woody biomass availability and cost, that, according on the demand for biomass in WITCH scenarios, contribute to the final cost of biomass (for more information see Emmerling et al. (2016)).

\subsection{Assumptions on CCS related parameters}

Most of the input data related to CCS power plants (such as costs, technical parameters and technological learning), have been updated or introduced in the model for the purposes of this study, after an extensive literature review. Follows an extract of this research, that highlights the main sources we referred to.

\subsubsection{CCS technologies overview}

According to (Metz et al., 2005), a $\mathrm{CO}_{2}$ capture system of a power plant can follow three possible layouts: post-combustion, pre-combustion and oxyfuel combustion. Each configuration can be applied to both fossil fuel and biomass combustion plants (Metz et al., 2005; IEAGHG, 2011a; ZEP/EBTP, 2012). CCS plants can moreover be built ex-novo or by means of retrofitting existing traditional plants. In terms of fuel use, different possible configurations are available. In this research, only coal, gas and biomass are considered as possible fuels, excluding other sources such as waste or mixed coal and biomass.

Pre-combustion capture of $\mathrm{CO}_{2}$ consists in separating the carbon dioxide before the fuel is 
burnt. The gas separation can be performed through gasification of fuels by means of steam reforming and water-gas-shift reactions, which produce syngas rich of hydrogen and $\mathrm{CO}_{2}$. Capture is then accomplished by an acid gas removal process of absorption and stripping with a chemical or physical solvent (GCCSI, 2012).

The post-combustion carbon capture technology aims to remove $\mathrm{CO}_{2}$ content from the stream of exhaust gases at the end of flue gases cleaning process. The separation of $\mathrm{CO}_{2}$ is usually performed using chemical solvents (i.e. MEA) or membranes. If required, this type of technology can be implemented on existing power plants (retrofitting), offering the opportunity to reduce plant emissions.

Oxyfuel power plants work with combustion of fuel with pure oxygen in a special boiler or furnace, usually with recirculation of exhaust gases (IEAGHG, 2010). The product of combustion after the exhaust treatment is mainly water and $\mathrm{CO}_{2}$. Water is easily condensed and separated, while $\mathrm{CO}_{2}$ is compressed and stored. Oxyfuel capture option is newer and less developed than pre- and post-combustion technologies (GCCSI/EPRI, 2012; Rubin et al., 2015). As a consequence, uncertainty on available data concerning oxyfuel is wider than for other technologies.

The introduction of climate policies is likely to result in the premature shutdown of existing fossil fuel plants (IEAGHG, 2011b). Retrofitting traditional plants to operate with CCS could therefore represent in some cases a cost effective alternative to a full replacement with lower carbon options (Gibbins et al., 2011; Johnson et al., 2015), despite the additional cost and the efficiency loss drawbacks.

In this study, we include four alternative coal-based CCS power plants: steam plants with post combustion, as new units (C-post) or obtained upgrading existing traditional plants (PC w/o CCS) with capture units (C-retro); a coal integrated gasification combined cycle with CCS (C-IGCC) and an oxyfuel plant with capture (C-OXY). We also consider a generic natural gas combined cycle with CCS (NG-CCS), which parameters are obtained averaging values related to different plant designs ${ }^{1}$. We similarly include a generic biomass-fueled power plant with capture (Bio CCS).

\subsubsection{Technical progress and associated impact on technology costs}

As CCS technologies are at an early stage of development, it is legitimate to hypothesize that technological improvements over time could impact on their cost and performance. In particular, a large body of literature advocates that an increase in installed capacity

\footnotetext{
${ }^{1}$ To be noted that the WITCH model aggregates single gas turbines and combined cycles without CCS under one single technology (NGCC w/o CC), while the NG-CCS technology only represents combined cycles with CCS.
} 
restrain the technology associated costs. One of the most diffused mathematical method for endogenously representing this phenomenon is through learning curves (Rubin et al., 2015; Jamasb, 2007). Often, literature reports the technology associated Learning Rate (LR), representing the reduction in cost corresponding to a doubling in installed capacity. The correlation between cumulative installed capacity and cost decrease is in this case represented by an exponentially decreasing curve. Even though the correlation is generally based on historical observations, Rubin et al. (2015) suggests a possible method for extending the concept of learning curves based on the learning of single technology subcomponents. This approach is functional for young technologies such as CCS power plants that lack empirical data (Wu et al., 2016; Li et al., 2012; Neij, 2008; Rubin et al., 2007). In this way, the modeler can apply a learning rate to the whole plant with CCS, as an average of single components learning rates, weighted on their costs. In this study, we gathered estimates and projections of learning rates and determined uncertainty ranges for each power plant category included in the model (see Table 2). Other technologies are likely to experience technological progress in the future: WITCH includes learning rates also for solar and wind plants. In this study we use the default values reported in Table 3 in the Appendix, without testing sensitivity ranges for these technologies.

\subsubsection{Technology related parameters of interest}

Tables 1 and 2 report the final results of our technological assessment in terms of nominal values and uncertainty ranges for each CCS technology considered in this study. Ranges are based on the arithmetic average of quantities reported from different sources. As an example, our nominal estimates (referred to as "best")is the mean of all the "best" estimates gathered from the references. When a single source provides ranges, we report them directly (i.e. Table 4). For a comparison of the data sources, we invite the reader to refer to Table 8 in the Appendix. Regarding Bio CCS, literature provides scarce and less reliable estimates concerning plants costs, efficiencies and learning rates. In this case we derive lower and upper bounds for each parameter consistently with the ranges obtained for the other CCS technologies.

Data gathered during this first phase allow to draw some preliminary insight on the current state of the art of CCS options in the power sector. First of all, values of capacity factor and electric efficiency seem comparable across coal fired plant technologies. Oxyfuel deviates from the others with its higher Capture Rate Ratio (CRR) and Investment Cost $\left(\mathrm{I}_{\text {cost }}\right)$, and a slightly lower Operating and Maintenance (O\&M) cost.

Secondly, the type of fuel has apparently important consequences both in terms of efficiency and costs. Natural gas combined cycle plants usually have higher efficiency and 
lower investment cost compared to coal plants (Beér, 2007). This holds true also for plants with carbon capture. Capture rate ratio, or capture efficiency, is instead comparable with each coal option (except for Oxyfuel).

Finally, Bio CCS shows in absolute terms the highest costs and lowest efficiency.

Looking at future perspectives, Oxyfuel is characterized by both the lowest learning rate and the narrowest range of uncertainty. This might appear to contrast with Oxyfuel being the currently least developed technology among the reported selection. However, the number of studies providing uncertainty assumptions for Oxyfuel learning rates is limited compared to other technologies, and the cost trend is mainly influenced by two expensive components, the Air Separation Unit (ASU) and the boiler, while other technologies have more heterogeneous cost drivers (IEAGHG, 2014b; MacDonald, 2012). This second aspect might influence the learning rate component based calculation.

Focusing on uncertainty ranges, Tables 1 and 2 show considerable variation in O\&M costs, investment costs and learning rates, while capture rates exhibit narrower ranges. Again, Oxyfuel departs from the others with more uncertain capital costs and more conservative electrical efficiency values.

Table 1: Main performance parameters of power plants with CCS as results of the literature review.

\begin{tabular}{lccccccccc} 
& \multicolumn{3}{c}{ Capacity Factor [\%] } & \multicolumn{3}{c}{$\mathrm{CO}_{2}$} & $\mathrm{CRR}[\%]$ & \multicolumn{3}{c}{ Net efficiency [\%] } \\
\cline { 2 - 11 } Technology & Low & High & Best & Low & High & Best & Low & High & Best \\
\hline PC w/o CC ${ }^{\text {b,c }}$ & & & 85 & & & & & & 45.0 \\
C-IGCC & 65 & 85 & 81 & 85 & 91 & 89 & 31.0 & 40.0 & 33.9 \\
C-post & 65 & 90 & 83 & 80 & 90 & 90 & 28.5 & 38.5 & 33.7 \\
C-OXY & 67 & 91 & 83 & 90 & 98 & 95 & 23.4 & 35.5 & 33.2 \\
NGCC w/o CC & & & 70 & & & & & & 50.0 \\
NG-CCS & 65 & 95 & 84 & 85 & 90 & 89 & 43.7 & 52.1 & 48.0 \\
Bio CCS & 65 & 84 & 80 & 85 & 91 & 90 & 0.2 & 0.36 & 28.0 \\
\hline
\end{tabular}

${ }^{\mathrm{a}}$ on LHV basis; $\quad{ }^{\mathrm{b}}$ Values currently used in the model, not output of literature review

${ }^{\mathrm{c}}$ same values for C-retro, with $\mathrm{CCR}=90 \%$ and $10 \mathrm{pp}$ efficiency loss

$\mathrm{d}$ includes single gas turbines still widely used globally, with low capacity factor

${ }^{\mathrm{e}}$ We assumed uncertainty ranges consistent with other CCS technologies

\subsubsection{Storage, transport and leakage}

Part of our modelling work has been aimed at including in WITCH costs, availability and reliability of carbon dioxide storage in geological reservoirs and transport through pipelines. Although this research focuses on the capture side of CCS, we underline that storage is a 
Table 2: Investments and O\&M cost of Power plants with and w/o CCS as results of the literature review (2005 USD).

\begin{tabular}{lrrrrrrrrr} 
& \multicolumn{1}{c}{$\mathrm{I}_{\text {cost }}[\$ / \mathrm{kW}]$} & \multicolumn{7}{c}{ O\&M costs $[\$ / \mathrm{MWh}]$} & LR [\%] \\
\cline { 2 - 9 } Technology & Low & High & Best & Low & High & Best & Low & High & Best \\
\hline PC w/o CC & & & 1472 & & & 8.9 & & & \\
C-IGCC & 1310 & 4648 & 3078 & 2.0 & 19.1 & 10.1 & 2.5 & 20.0 & 6.7 \\
C-post & 1400 & 4627 & 3063 & 3.8 & 22.8 & 13.4 & 1.1 & 9.9 & 3.8 \\
C-OXY & 1127 & 5034 & 3253 & 1.7 & 20.5 & 9.5 & 1.4 & 7.0 & 2.8 \\
C-retro & & & 2302 & & & 13.4 & & & \\
NGCC w/o CC & & & 750 & & & 5.6 & & & \\
NG-CCS & 774 & 2268 & 1508 & 4.6 & 8.3 & 6.4 & 1.2 & 12.0 & 4.2 \\
Bio CCS & 1662 & 5540 & 3693 & 3.1 & 20.6 & 10.3 & 0.0 & 10.0 & 5.0 \\
\hline
\end{tabular}

${ }^{a}$ Values currently used in the model, not output of literature review

b Summed costs of old power plants and retrofitting unit

${ }^{\mathrm{c}}$ We assumed uncertainty ranges consistent with other CCS technologies

potential driver of uncertainty in long-term projections and consider it an important avenue for future research.

Nowadays, $\mathrm{CO}_{2}$ transport and storage technologies are mature and mainly applied to Enhanced Oil Recovery (EOR) $\mathrm{CO}_{2}$ supply. Particularly in the United States, $\mathrm{CO}_{2}$ from natural sources has been injected for decades into expiring oil fields for enhancing the extraction (IEA, 2015; Godec et al., 2011).

Most studies agree to consider a set of storage option: depleted or remaining oil and gas fields, coal beds and underground saline aquifers, not used for drinkable water.

For this work we derived an average storage capacity for each region in the model and for seven different types of storage reservoir, including onshore and offshore options. Any detail concerning the set of assumptions we adopt to model storage sites are reported in the Appendix.

Regarding transportation of $\mathrm{CO}_{2}$, the most diffuse technique is liquefaction and conveyance through pipelines. Although some other options are available, mainly ships transport, they prove not to be economically convenient except for particular cases (i.e. remote offshore distances) (ZEP, 2011b).

$\mathrm{CO}_{2}$ transportation costs via pipeline are mainly related to the infrastructure costs, followed by costs of allowances, surveillance and expert supervision (McCoy and Rubin, 2008). Our implementation introduced an average distance between storage site and power plant with CCS per each type of storage and region, that, combined with an average transportation cost per $\mathrm{km}$, provides the cost of $\mathrm{CO}_{2}$ transport. 
An important aspect of $\mathrm{CO}_{2}$ storage and transportation is represented by leakage risk. The term leakage refers to undesired $\mathrm{CO}_{2}$ losses due to imperfect sealing or infrastructures damages. Leakage could be originated by $\mathrm{CO}_{2}$ transportation, underground injection, or storage and it might significantly hinder the benefits of CCS (van der Zwaan and Smekens, 2009). For the purpose of this analysis, as a first approximation we consider leakage rates equal to zero. We refer to the IPCC, 2005 report, according to which storage sites are highly probably reliable and safe, meaning releasing practically no leakages (Metz et al., 2005). However, given the high uncertainty related to leakage estimates and sites monitoring (Bielicki et al., 2015), the Authors explored the implications of leakage and its uncertainty in a different study, under the same modelling framework used in this research (Vinca et al., 2018).

\subsection{Description of the analysed scenarios}

In this study, climate policy scenarios are identified by temperature targets, i.e. a limit on temperature increase with respect to the preindustrial level over the century. This constraint can be conveniently added to the WITCH model in the form of a cap on cumulative GHG emissions (carbon budget). The higher the carbon budget, the higher the temperature increase, and the lower the associated policy stringency. The model iteratively searches for a global price on anthropogenic GHG emissions that eventually achieves the desired carbon budget. This price is implemented as a carbon tax that comes into force in 2020 and rises over time at the interest rate of the global economy.

In order to have a more reliable estimate of the resulting temperature pathways, we take advantage of the soft link between WITCH and the MAGICC climate model (Meinshausen et al., 2011). MAGICC receives as input the GHG emission levels obtained by WITCH and provides values of average global temperature increase, radiative forcing and average concentration of $\mathrm{CO}_{2}$ in the atmosphere. MAGICC produces a reference temperature pathway leading to a certain temperature increase in 2100: this baseline corresponds to the median of temperature increase distribution, and represents therefore a scenario with $50 \%$ of probability of meeting a certain temperature target. In this study, we adopt a conservative approach: instead of considering the median of the distribution as a baseline, we use as reference pathways where the probability of meeting a certain temperature target exceeds $60 \%$. We name each scenario after the temperature increase resulting from this procedure (i.e. if WITCH emissions pathway output determines a MAGICC $1.5{ }^{\circ} \mathrm{C}$ temperature increase with a probability exceeding $60 \%$, the scenario is called $1.5 \mathrm{DC})$.

We consider several scenarios imposing different carbon budgets on overall GHG emissions, 
reporting commonly used metrics such as the $\mathrm{CO}_{2}$ emissions from 2010 to 2100 from anthropogenic sources, or the mean global temperature increase. As already underlined, we are particularly interested in analyzing scenarios in line with the Paris agreement. We therefore investigate in greater detail two representative scenarios, called $1.5 \mathrm{DC}$ and $2 \mathrm{DC}$ according to our notation. 1.5DC scenario imposes a $\mathrm{CO}_{2}$ budget from 2010 to 2100 of around $500 \mathrm{GtCO}_{2}$, with a $60 \%$ chance of containing warming below $1.5^{\circ} \mathrm{C}$. In the $2 \mathrm{DC}$ scenario, the budget by the end of the century is less than $1200 \mathrm{GtCO}_{2}$, with a temperature increase in 2100 lower than $2^{\circ} \mathrm{C}$ with a $60 \%$ chance. These budgets are in line the standardized scenarios highlighted by the IPCC AR5 report (IPCC, 2014) as Representative Concentration Pathways (RCP) 1.9 and 2.6 respectively for 1.5DC and 2DC scenarios (Vuuren et al., 2017). It should be noted that the carbon budgets here selected lie slightly above the average values in the ranges set by the IPCC. Finally, we also explore scenarios associated with larger budgets (more details are shown in Figure 6 in the Appendix).

\subsection{Robustness checks on the input parameters}

Due to the scarce penetration of CCS in the current power fleet and the uncertainty of its future development, we perform a robustness check on the associated cost and performance assumptions. In particular, we focus on six parameters: plant capacity factor, net efficiency, capture rate ratio, investment and operation and maintenance costs and learning rates (for a description of the learning rate, see Section 2.2.2). For the selected scenarios (1.5DC and 2DC), we perform a sensitivity analysis on each of these parameters in turn (ceteris paribus) to test how the model output changes in case of nominal, low and high estimates. This kind of analysis puts in evidence which parameters are most notably affecting outcomes in terms of installed CCS power capacity. A further robustness check addresses the third research question. In particular, once the most significant parameters are identified by the procedure above, we extend the sensitivity analysis to additional scenarios with different carbon budgets. We consider temperature increase targets between 1.5 to $4^{\circ} \mathrm{C}$, the latter being consistent to our BAU case. This will reveal whether the response to the parameters variation have different impacts on the outcomes according to the target.

\section{Results}

In the following Section, we introduce the reference results, i.e. those obtained with nominal

values for the parameters of interest, under the 1.5DC and 2DC scenarios. For the same scenarios, we further perform a parametric uncertainty analysis. We conclude extending the 
study of CCS deployment under a wider range of policies.

\subsection{CCS in 1.5DC 2DC scenarios}

\subsubsection{Reference results}

Figure 1 shows the amount of electricity generated by CCS plants over time in the 2DC (upper panel) and 1.5DC (lower panel) scenarios (the full energy mix is reported in the Appendix). Considering the 2DC case, retrofitting existing coal power plants seems a cost effective choice just after the carbon tax introduction, for a few decades (until 2050). A first peak in CCS deployment occurs before 2050, followed by a decrease driven by the retirement of retrofitted capacity and a shift towards renewable energy technologies. Considering the full electricity mix, CCS plants contribute to $8 \%$ of the yearly electricity generation in 2050, compared to around $16 \%$ of nuclear and $73 \%$ of wind solar and hydroelectric technologies. In the long run, CCS production increases again as investments are focused on Bio CCS, the only technology in the model capable of achieving negative emissions. If we compare our results (after the redesign of power-CCS we performed for this study) with WITCH prior projections, we notice that the obtained shares of fossil fuel CCS and bio CCS are similar. However, our results show a major contribution of coal with CCS (see Koelbl et al. (2014) also for multi-model comparison on CCS in $2^{\circ} \mathrm{C}$ scenario). Concerning non-retrofitted coal fueled options, Oxyfuel seems the most promising technology, despite its high costs and limited learning improvement. Yet its high capture rate compensates for such drawbacks, especially in the context of a rather stringent scenario like 2DC.

Looking at 1.5DC, we notice an almost total absence of fossil fueled CCS plants, while negative emission technologies gain significant importance growing steadily until 2100 . We point out that due to the capture rates lower 100\%, both fossil and biomass fueled plants with CCS emit a small amount of carbon into the atmosphere. Considering Bio CCS however, net $\mathrm{CO}_{2}$ emissions are negative. As opposed to 2DC, 1.5DC does not leave room for net emitting technologies, so that Bio CCS, renewables and nuclear are the preferred low carbon alternatives (respectively 4\%,80\% and $16 \%$ of the electricity generation in 2050). As already specified, the contribution of Bio CCS becomes increasingly relevant approaching 2100, when it accounts alone for $11 \%$ of the electricity generation.

Use of Bio CCS in electricity and other sectors leads to global use of woody biomass around $100 \mathrm{EJ} / \mathrm{yr}$ in 2050 and $220 \mathrm{EJ} / \mathrm{yr}$ in 2100. Being biomass availability set by the GLOBIOM model respectively to 180 and $235 \mathrm{EJ} / \mathrm{yr}$ in 2050 and 2100, our results for the 1.5DC scenario are getting close to the availability constraint. The magnitude we obtain is in line with assumptions and results from (Heck et al., 2018; Mander et al., 2017). In 


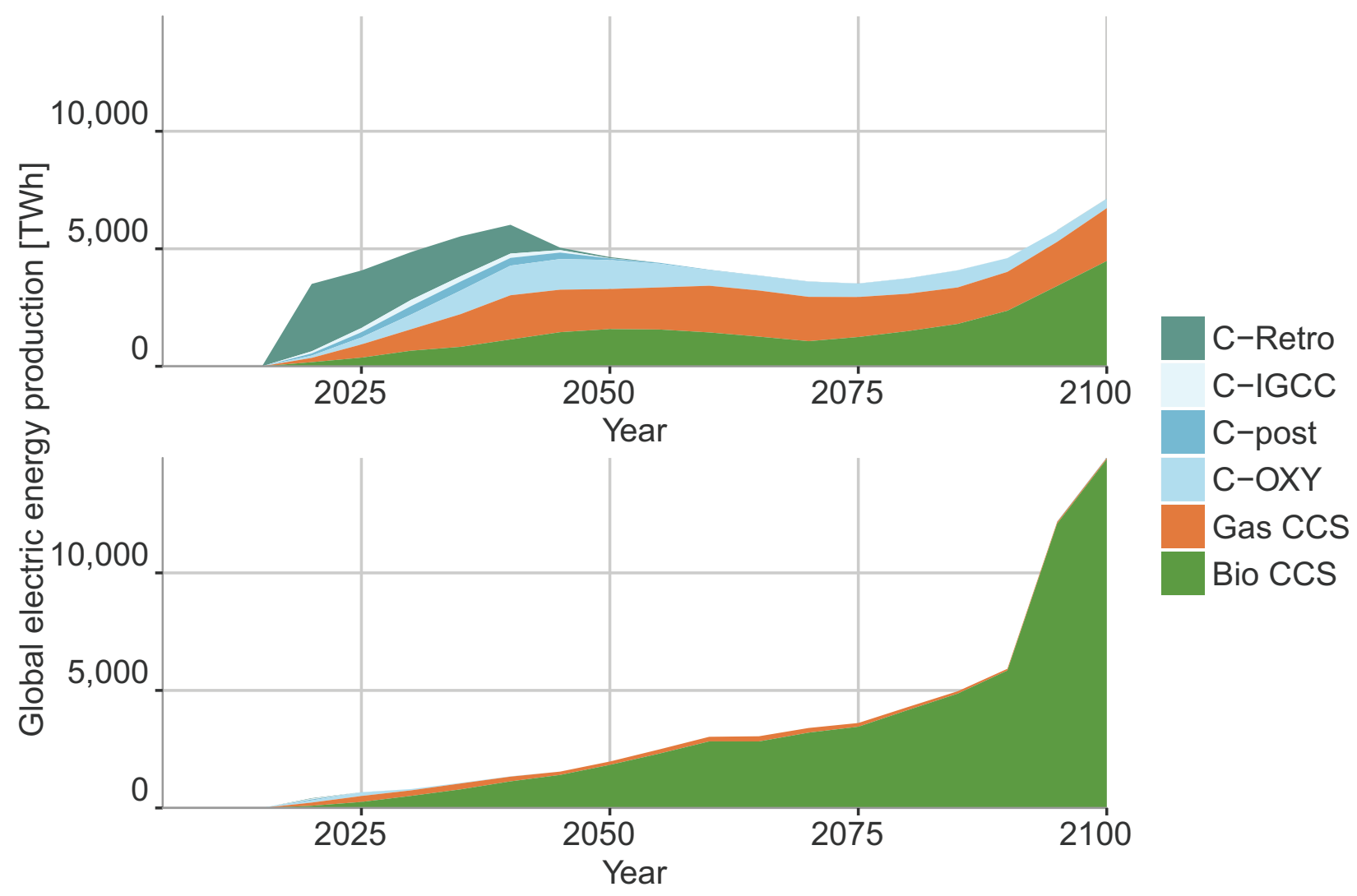

Figure 1: Electricity generation mix by CCS plants over time, for the 2DC (upper panel) and the $1.5 \mathrm{DC}$ (lower panel) scenarios 
contrast, our results show minor use of biomass both in the electricity and fuel sector than in studies performed with the GCAM IAM (considering the 2DC scenario only) (Muratori et al., 2016, 2017). As can be noticed from other studies, assumptions in WITCH related to biomass and other renewables lead to scenario with high use of solar and wind as mitigation technologies (Luderer et al., 2017). As for captured $\mathrm{CO}_{2}$ storage, available capacity seems not to be a binding constraint. In both scenarios, CCS power plants captured emissions suffice a remarkably small portion of the available storage capacity. By the end of the century, they account for less then $5 \%$ of the nowadays estimated available capacity: around $460 \mathrm{GtCO}_{2}$ (2DC) and $470 \mathrm{GtCO}_{2}(1.5 \mathrm{DC})$ out of more than $11000 \mathrm{GtCO}_{2}$ available at a global level. However, some regions use a substantial share of their respective storage capacity: e.g. up to $45 \%$ (China), or 25\% (South America).

\subsubsection{Parametric uncertainty}

In order to test the model response to different input estimates, we analyzed how the installed capacity of CCS power plants changes in both 2DC and 1.5DC scenarios using minimum and maximum values for the parameters of interest as reported in Tables 2 and 1 . Results are shown in Figure 2, where bars represent the percentage variation in cumulative installed capacity of all CCS plant categories with respect to the nominal case, which corresponds to the central black line. Measures span periods ranging from 2015 to 2050 and from 2015 to 2100. For each parameter, the results corresponding to the maximum value (referred to as "High" in the tables) is marked with a dot.

In general, efficiency, capture rate and investment cost lead to broader deviation in the output, whereas learning rate, capacity factor and O\&M cost almost always cause variation lower than $10 \%$.

Among others, the impact of capture rate ratio is particularly remarkable considering that this parameter has the narrowest uncertainty gap. Its effect grows over time for the 2DC scenario, producing variation from $-15 \%$ to $+12 \%$ on the installed CCS capacity. An opposite trend holds for the 1.5DC scenario, where Bio CCS is extensively used early in the century to achieve global negative emissions, and an extreme realization of CRR levels can either significantly interfere or align with this goal.

The second most influential parameter, at least for the 2DC case, is the investment cost. Higher CCS capital costs make the construction and use of this type of plants less preferable, as other available low-carbon options keep their nominal costs. The response is weaker in the more stringent case. The latter involves higher policy costs, against which capital cost variations impact relatively little. Similar results hold for the other monetary parameter of the group, namely O\&M costs, but with a minor response, as installation costs dominate 


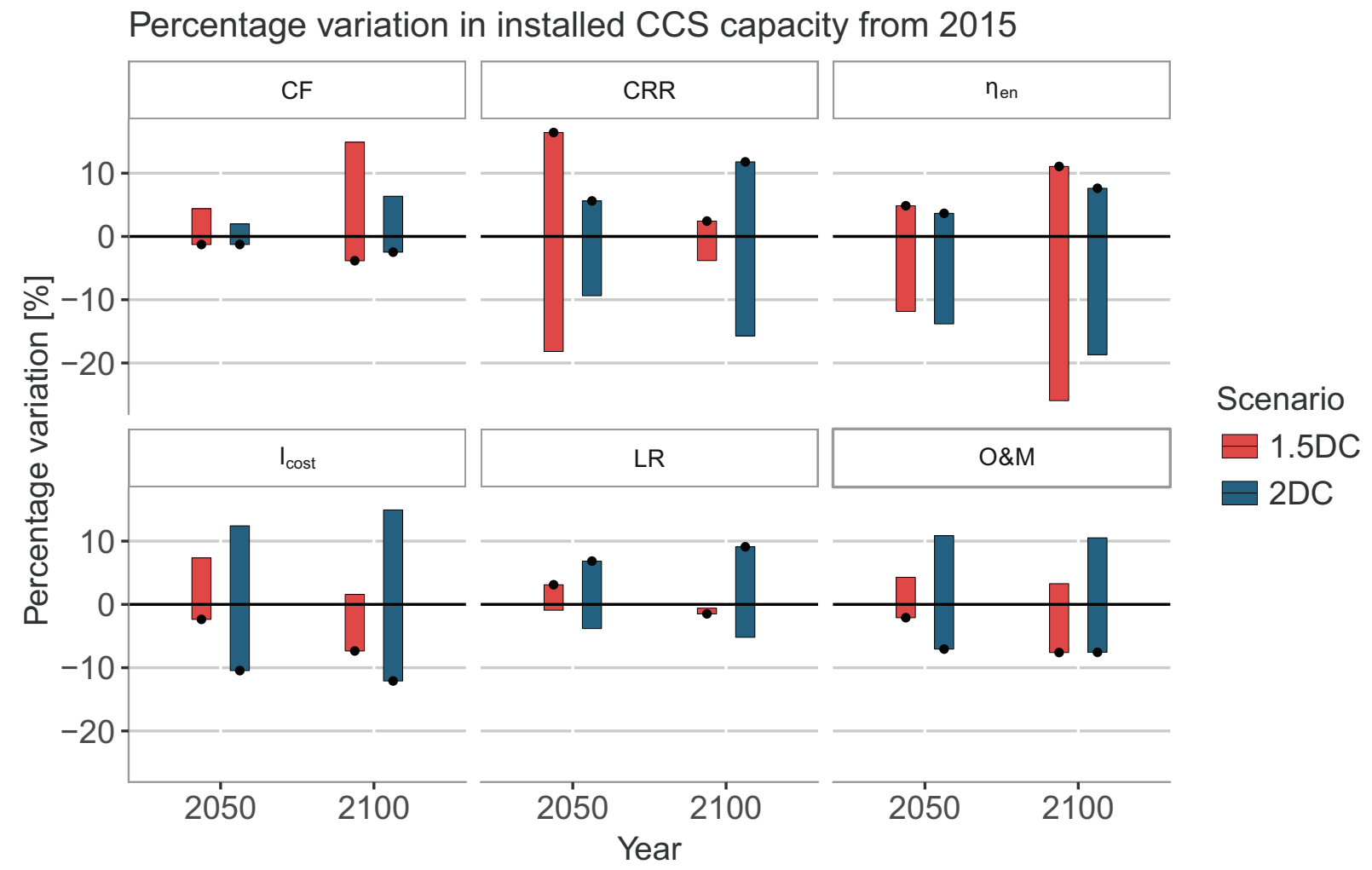

Figure 2: Percentage variation in cumulative installed CCS capacity in response to changes in six performance parameters 
operation costs for these types of plant.

Considering electric efficiency, we notice how a low value is particularly detrimental for CCS development in both climate constrained scenarios, showing a tumble of $-26 \%$ for $1.5 \mathrm{DC}$ in 2100. Efficiency links energy production with plant emissions, so that a lower value implies higher $\mathrm{CO}_{2}$ emissions per unit of energy produced, reducing the mitigation benefits of CCS. On the other hand, higher efficiencies can translate into either keeping the same amount of energy with less associated emission, or producing more energy (and installing more plants) while keeping the same absolute level of emissions. Our model suggests the latter to be the most convenient strategy.

Although the capacity factor establishes the relation between installed capacity and electricity generation (hence the amount of captured emissions), the variation in this parameter does not affect particularly the stock of CCS installed. Either its uncertainty range or its unitary impact is smaller with respect to the others, with the exception of 1.5DC by 2100 . In this case, higher stress is put on the yearly generation of large capacities of biomass plants to keep global cumulative emissions below the budget, and the sensitivity is higher.

Effects of alternative assumptions in technological improvement seem also small with respect to the others. Learning rate shares similar trends across time and policy stringency with the other two monetary metrics, as it is intimately related to costs in our implementation. The impact is negligible in the $1.5 \mathrm{DC}$ case, where the range of variability does not seem to justify a change in a strategy already with little room for deviations over time.

To sum up, uncertainty in investment costs drives changes in CCS development the most under a $2^{\circ} \mathrm{C}$ temperature increase target, while costs becomes less relevant for 1.5 degrees, and the uncertainty on technological parameters affecting emissions are more critical. In this latter case, cost assumptions do matter for the overall policy costs, but not as much in determining the optimal CCS strategy. The robustness check towards investment cost is in line with our sources (Muratori et al., 2016), but in addition we highlighted how efficiency or capture removal become determinant in the most stringent scenarios. Finally, comparing 2050 to 2100 metrics, the impact of parameter variability tends to grow over time, with the most notable exception of CRR under 1.5DC.

\subsection{CCS deployment across different temperature targets}

In this section we consider how CCS utilization changes across a continuum of temperature targets. The most stringent scenario is $1.5 \mathrm{DC}$, and the most lenient one corresponds to an average temperature increase of around $4^{\circ} \mathrm{C}$ in 2100 , which is the climate outcome of a BAU.

Figure 3 shows the cumulative electric energy produced by CCS plants up to 2100 in our 
range of scenarios. At the extreme right we have a BAU output, where no CCS generation is needed. Going towards the left, climate stringency increases, and CCS energy production starts to be positive for targets below $3.4^{\circ} \mathrm{C}$. As temperature target falls, we notice a monotonic increase in energy production, for each type of CCS plant, down to around $2.5^{\circ} \mathrm{C}$. As we go further towards the left-hand side of the chart, total energy from CCS plants lowers, while retrofitting gains importance to the detriment of Bio CCS. In these scenarios, the level of carbon price at the introduction of the tax is high enough to make C-Retro highly competitive, triggering a fast reduction in emission before 2050, with Bio CCS entering the energy mix later in time (see Figure 1). The abatement provided by retrofitting is enough to reduce the need for Bio CCS negative emissions. For achieving very low targets (temperature lower than $2^{\circ} \mathrm{C}$ ), we see a strong reduction in electricity production from fossil fuel plants, which follows a general reduction in total energy supply. Nonetheless, Bio CCS increases significantly, as it is the only technology that provides negative emissions.

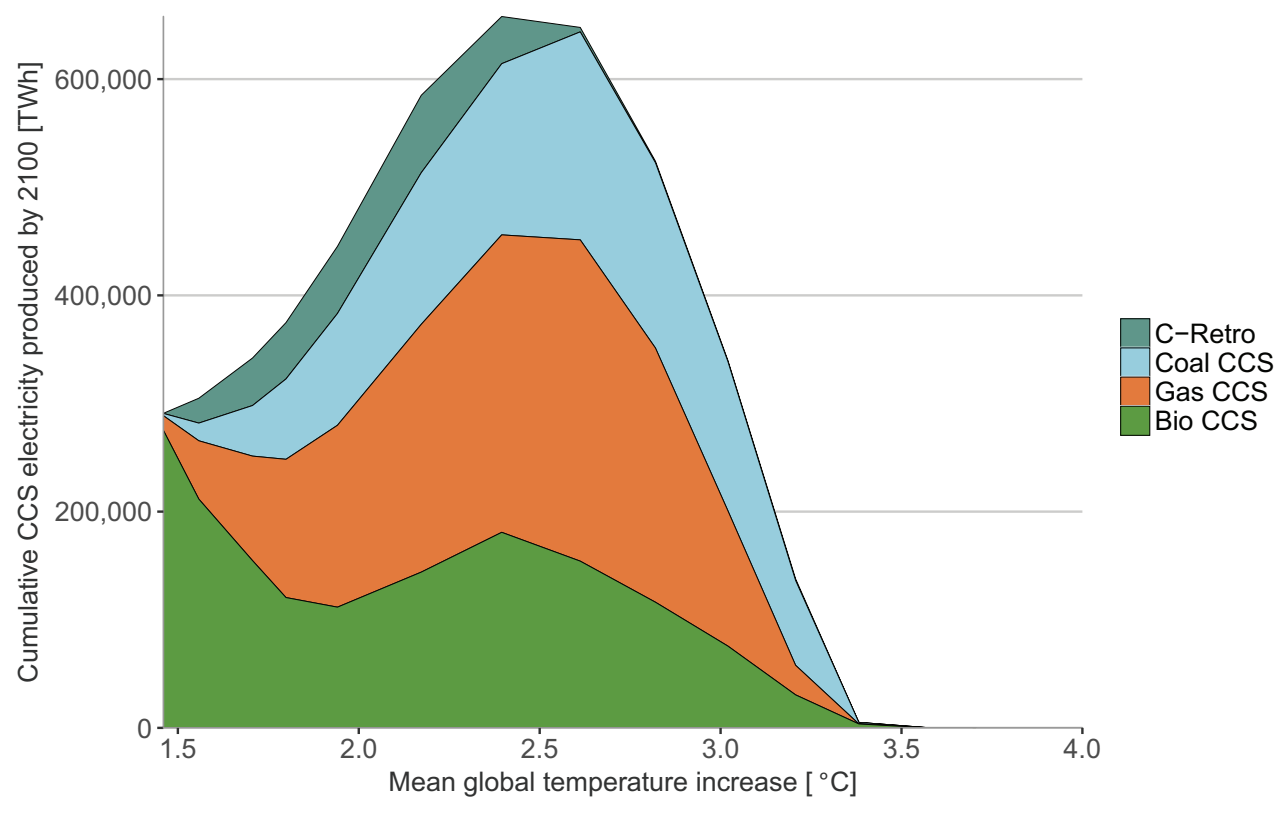

Figure 3: Cumulative electricity generation from CCS plants by 2100 for different temperature increase targets

Thus, fossil fuel fired plants with CCS seem optimal for achieving targets of medium to medium-high stringency, around $2.5^{\circ} \mathrm{C}$. In order to limit the temperature increase below $1.5^{\circ} \mathrm{C}$ from pre-industrial level with mitigation measures alone, biomass with CCS is the most economically efficient alternative. In this case, the total amount of CCS electricity production depends mostly on biomass availability and cost.

Figure 4 shows the total CCS installed capacity up to 2100 for different climate scenarios, and how this deviates from the nominal case (black dotted line) when considering either high 
or low efficiencies and capital costs. The impact of varying climate policy dominates the one of alternative parameterizations, which share similar concave curves with a peak around $2.5^{\circ} \mathrm{C}$. As confirmed by our previous analysis, the variation in investment costs determines the greatest change in output for medium-stringent scenarios. Moving below $2^{\circ} \mathrm{C}$, the outcomes of alternative costs converge, while the impact of efficiency becomes more pronounced.

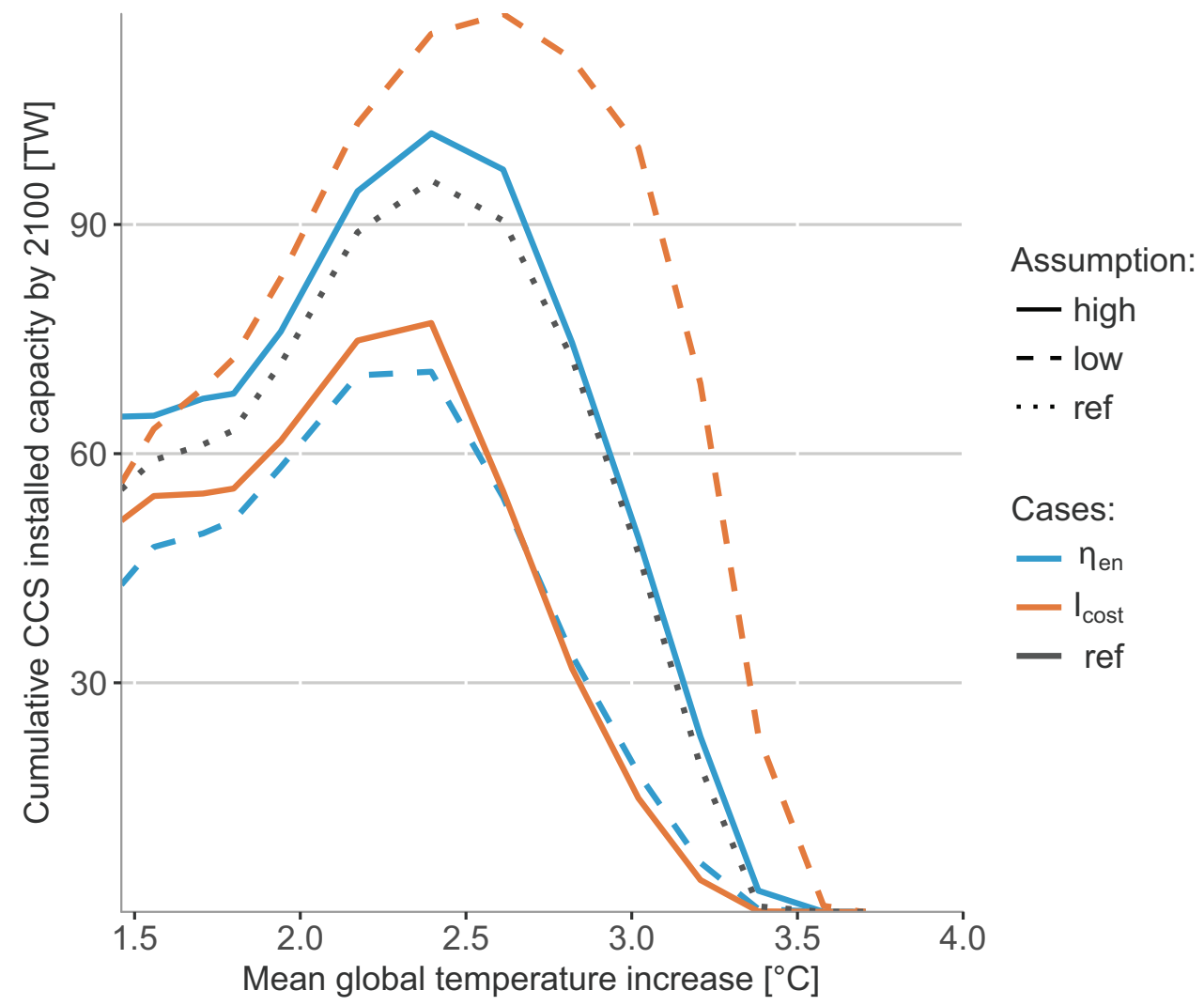

Figure 4: Variation in installed CCS capacity over temperature increase, in response to changes in efficiencies and investment cost

Finally, Figure 5 compares the share of the yearly global electricity generation for CCS and renewable technologies with intermittences (solar and wind). This analysis considers all the different temperature targets examined in Figure 3 and three time steps, 2030, 2050 and 2100. First of all, the bell shape behaviour of CCS share across climate policies emerges in these charts. By contrast, renewables follow a continuous increase as the temperature target tightens, exhibiting complementarity with CCS options. This trend stands true over time, with renewables becoming predominant in the energy mix moving towards the end of the century. The 2100 related curve presents some differences compared to the others. First of all, the maximum share of CCS considering all the scenarios is around 10\%, while in 2030 and 2050 it exceeds 15\%. Moreover, in 2100 and in case of very stringent scenarios, Bio-CCS 
grows significantly, while the share of renewables slightly drops and remains below $80 \%$.

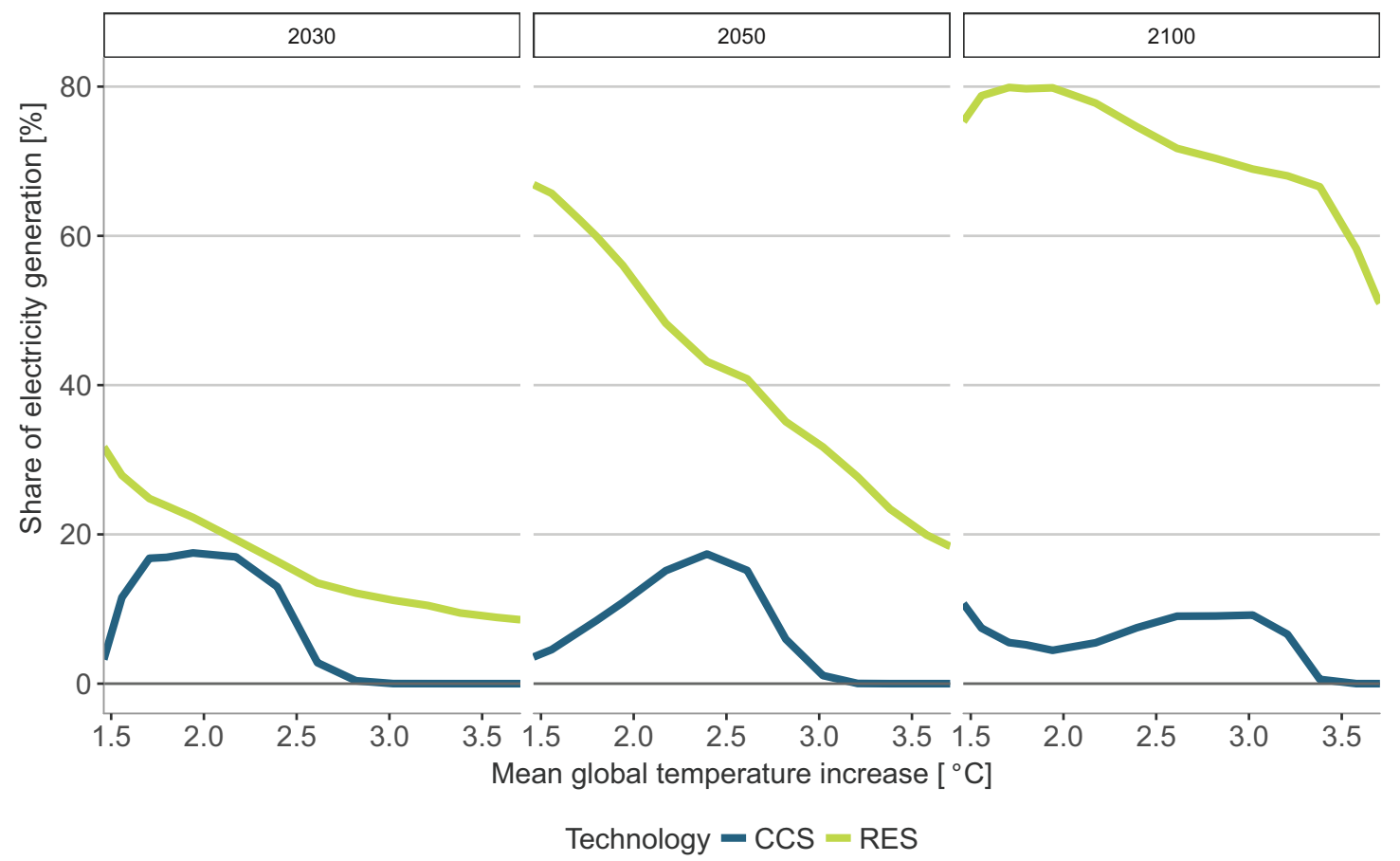

Figure 5: Comparison of CCS and intermittent RES share of yearly total electricity generation, across different climate targets.

\section{Conclusions}

With this study, we aim at contributing to the debate about fossil CCS potential as a low carbon technology in medium- to long-term scenarios, when considering techno-economic and policy uncertainties. We provide at first an overview of CCS plant available options and we later explore different scenarios with the WITCH model. Our analysis spans a range of temperature targets, with a focus on scenarios consistent with the Paris Agreement. Results indicate that:

1. CCS technological portfolio currently available covers a range of multiple alternatives, both in terms of energy source (coal, gas, biomass) and conversion system (pre- and post-combustion, oxyfuel combustion). These aspects impact on technical parameters associated values, and affect as a consequence the technologies potential. All technologies results competitive in some important scenarios (e.g. 2DC). In particular, due to high capture rate ratio, oxyfuel coal plants seem the most promising alternative among coal technologies, despite the high cost. Retrofitting existing plants also plays and important 
role, as well as Bio CCS, which finds the same share in the electricity mix as natural gas with CCS, even if plant associated costs are roughly twice as much. Bio CCS remains however the uncontested best option in most stringent scenarios (1.5DC).

2. Efficiency, capture rate and investment cost lead to broader deviation in installed capacity of CCS options, whereas learning rate, capacity factor and O\&M cost have marginal impact. Sensitivity to plant characteristic parameters is mainly influenced by costs in medium-stringent scenarios while efficiencies prevail at lower carbon budgets, where only Bio CCS seems a competitive technology. Time seems to be a determinant factor when analyzing the sensitivity, as sensitivity appears to be more significant in the long run. In the medium-stringent scenarios however, as an early strong mitigation is introduced by retrofitting, this dependency appears to be less evident.

3. Fossil fuel fired plants with CCS are preferably deployed in medium to medium-high stringency scenarios (temperature target around $2.5^{\circ} \mathrm{C}$ ). More ambitious targets require instead large biomass CCS deployment, to the detriment of fossil CCS.

\section{$5 \quad$ Limitations and further developments}

Even though in our understanding this research provides some valuable insights on CCS perspectives, we acknowledge some major limitations.

First of all, even if the modeling phase has implied a careful representation of both capture and storage processes of CCS technologies, in the results we exclusively focus on the capture side, neglecting the significant sensitivity of the output with respect to storage and leakage related parameters. We addressed this issue in another other study, providing an analysis on storage costs and capacity and leakage values and trends. Other applications of CCS technologies are also overlooked in the current model version, namely carbon capture in industrial applications and CCS in the production chain of secondary energy carries other than electricity (hydrogen and synfuels). These aspects, together with carbon utilization, could be included in the model to improve the current carbon capture and storage representation.

\section{Appendix}

\section{Additional input and output of the WITCH model}

Figure 6 shows the mean temperature increase for the adopted scenario, output of the MAGICC climate model having in input the WITCH GHG emission levels. . 


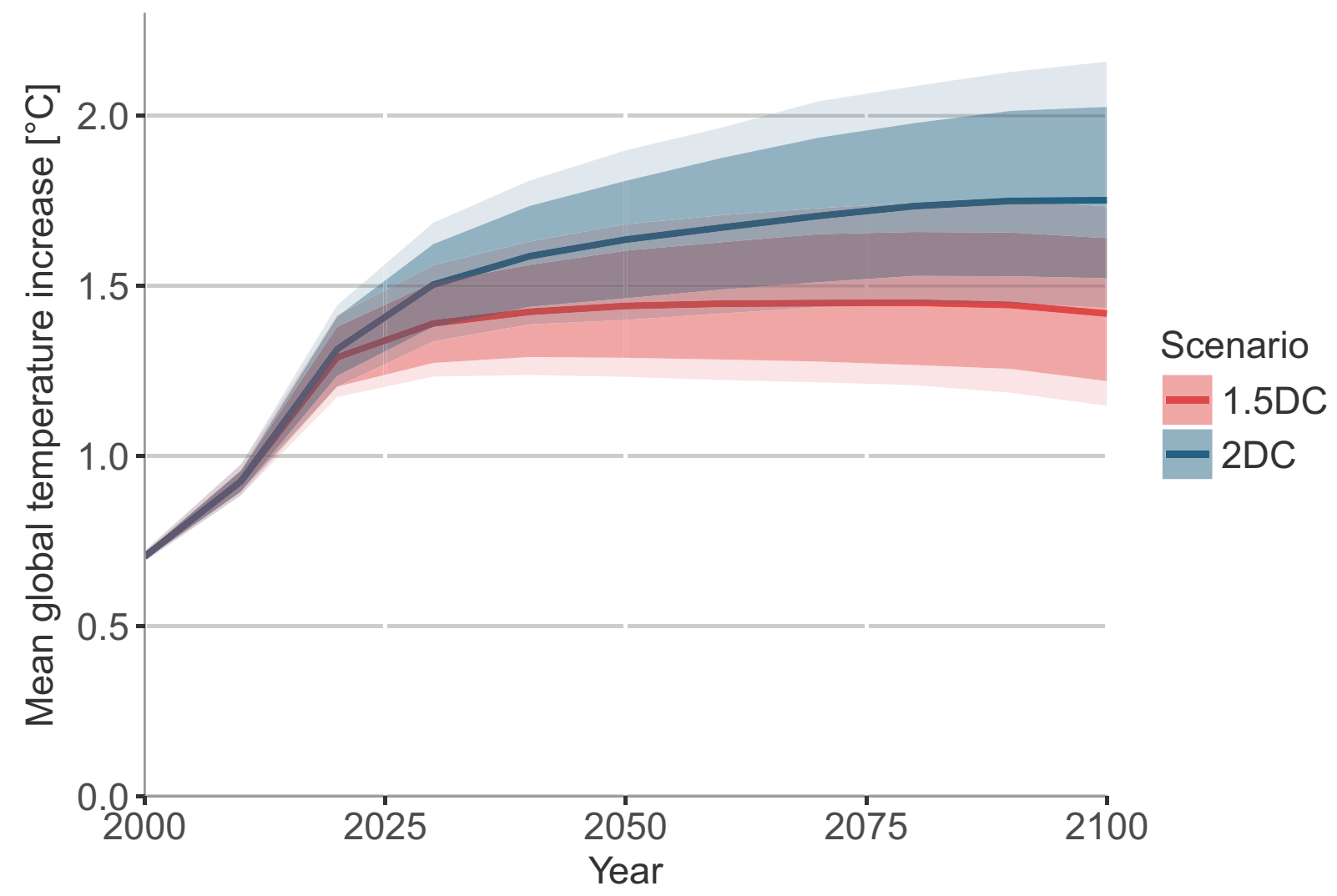

Figure 6: Average temperature increase for 2DC and 1.5DC scenarios with two confidence interval areas. The dark shading denotes the $25 \% / 75 \%$ percentile region, and the light shading the $17 \% / 83 \%$ percentile region. The lines in the middle represent the median. 
Figure 6 shows the electricity generation mix showing the whole technological portfolio included in WITCH.

Wind
Solar
Oil
Nuclear
Hydro
Natural Gas
Coal
C-Retro
Coal CCS
Gas CCS
Bio CCS
Biomass

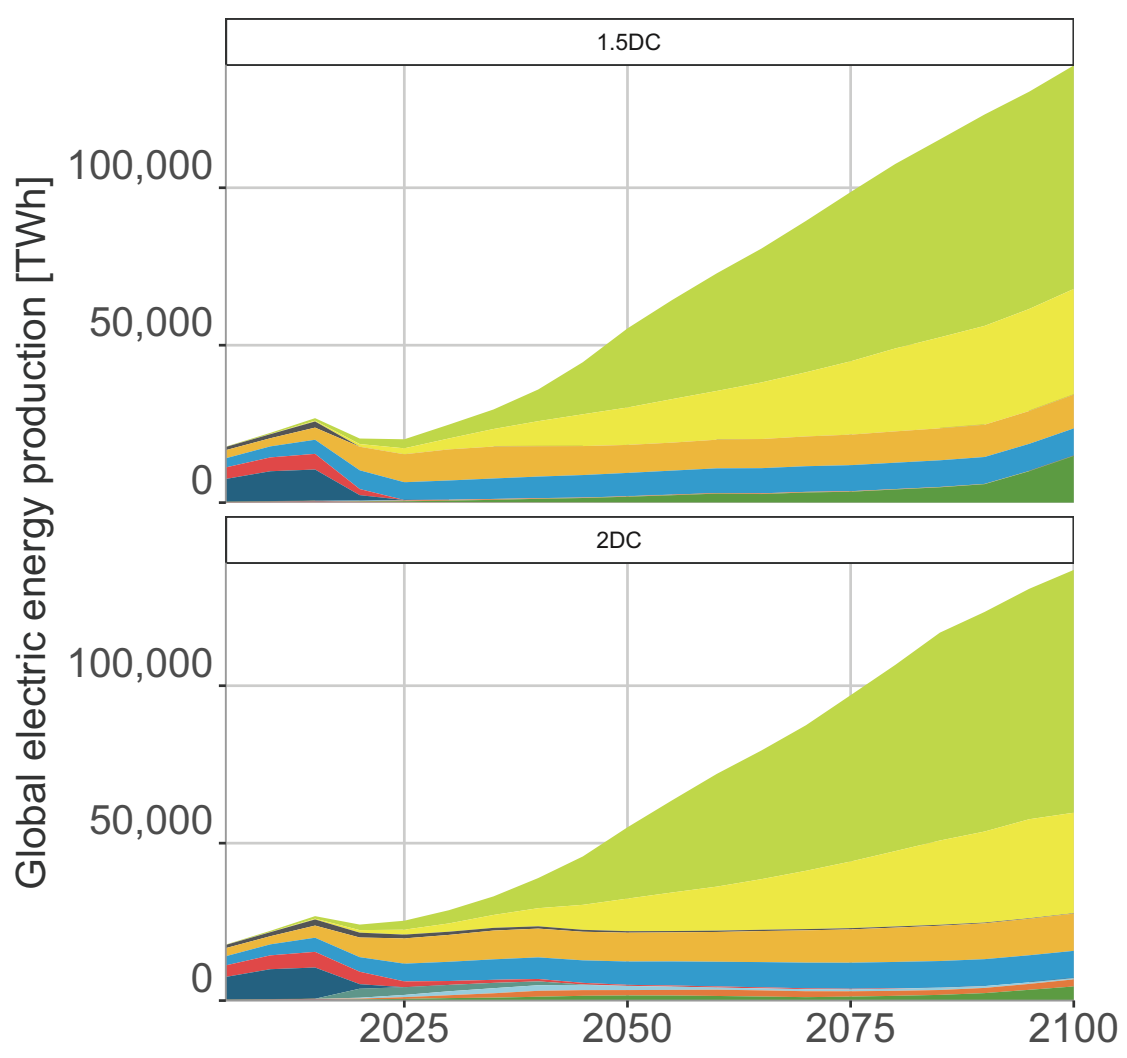

Figure 7: Electricity generation mix for 1.5DC and 2DC scenarios.

Table 3: Renewable energy technologies input data. These are representative of the Western Europe region, values differ regionally. Sources (Neij, 2008; Kaltschmitt et al., 2007)

\begin{tabular}{llll} 
plant & LR [\%] & $\mathrm{I}_{\text {cost }}[\$ / \mathrm{kW}]$ & Capacity Factor [\%] \\
\hline elpv & $17 \%$ & 4650 & $26 \%$ \\
elcsp & $10 \%$ & 6120 & $41 \%$ \\
elwindon & $10 \%$ & 1470 & $20 \%$ \\
elwindoff & $13 \%$ & 2640 & $36 \%$ \\
\hline
\end{tabular}

\section{Data from literature review}


Table 4: $\mathrm{CO}_{2}$ storage capacity divided per region in low, best, high cases in $\left[\mathrm{GtCO}_{2}\right]$ (IEAGHG, 2011a).

\begin{tabular}{lrrrrrrrrr} 
& \multicolumn{3}{c}{ O\&G } & \multicolumn{3}{c}{ Coal beds } & \multicolumn{3}{c}{ Aquifers } \\
\cline { 2 - 10 } & low & best & high & low & best & high & low & best & high \\
\hline Africa and ME & 209 & 522 & 1430 & 0 & 8 & 46 & 216 & 588 & 1736 \\
Asia & 36 & 91 & 234 & 0 & 179 & 967 & 53 & 370 & 1614 \\
Oceania & 8 & 20 & 49 & 0 & 11 & 54 & 0 & 2 & 9 \\
Latin America & 29 & 89 & 331 & 0 & 2 & 12 & 33 & 121 & 479 \\
non OECD \& ex USSR & 310 & 310 & 310 & 25 & 25 & 25 & 379 & 379 & 379 \\
North america & 22 & 156 & 166 & 157 & 176 & 229 & 3307 & 8001 & 12774 \\
OECD Eu & 19 & 19 & 19 & 1 & 1 & 1 & 82 & 82 & 82 \\
\hline
\end{tabular}

Table 5: Percentage of onshore and offshore capacity for storage potential of O\&G and Aquifers (IEAGHG, 2009).

\begin{tabular}{lrrrr} 
& \multicolumn{2}{c}{ O\&G } & \multicolumn{2}{c}{ Aquifers } \\
\cline { 2 - 5 } & onshore & offshore & onshore & offshore \\
\hline Canada & $95 \%$ & $5 \%$ & $80 \%$ & $20 \%$ \\
USA & $80 \%$ & $20 \%$ & $75 \%$ & $25 \%$ \\
Latin America & $40 \%$ & $60 \%$ & $80 \%$ & $20 \%$ \\
Eastern Europe & $25 \%$ & $75 \%$ & $35 \%$ & $65 \%$ \\
Former SU & $75 \%$ & $25 \%$ & $20 \%$ & $80 \%$ \\
Middle East & $75 \%$ & $25 \%$ & $95 \%$ & $5 \%$ \\
India & $65 \%$ & $35 \%$ & $50 \%$ & $50 \%$ \\
China & $95 \%$ & $5 \%$ & $80 \%$ & $20 \%$ \\
Australia and New Zealand & $0 \%$ & $100 \%$ & $30 \%$ & $70 \%$ \\
South East Asia & $20 \%$ & $80 \%$ & $40 \%$ & $60 \%$ \\
Western Europe & $20 \%$ & $80 \%$ & $35 \%$ & $65 \%$ \\
Africa & $35 \%$ & $65 \%$ & $35 \%$ & $65 \%$ \\
\hline
\end{tabular}

Table 6: Cost of storage for O\&G sources and Saline formations [\$/ $\left.\mathrm{tCO}_{2}\right]$ (ZEP, 2011a).

\begin{tabular}{lllrrr} 
& & & low & high & best \\
\hline \multirow{4}{*}{ Onshore } & Depleted O\&G & reuse & 1.28 & 8.98 & 3.85 \\
& Depleted O\&G & no reuse & 1.28 & 12.83 & 5.13 \\
& Saline formations & & 2.57 & 15.39 & 6.41 \\
& Depleted O\&G & reuse & 2.57 & 11.54 & 7.70 \\
\multirow{2}{*}{ Offshore } & Depleted O\&G & no reuse & 3.85 & 17.96 & 12.83 \\
& Saline formations & & 7.70 & 25.65 & 17.96 \\
\hline
\end{tabular}


Table 7: Transport costs on a common basis for onshore and offshore pipelines at three different capacities $\left[\$ / \mathrm{tCO}_{2} / 250 \mathrm{~km}\right]$ (Rubin et al., 2015). Original sources are reported in the first column: the values have been converted in the same unit of measure by the author to ease the comparison.

\begin{tabular}{lrrrrrr} 
& \multicolumn{3}{c}{ ONSHORE } & \multicolumn{3}{c}{ OFFSHORE } \\
\cline { 2 - 7 }$\left[\mathrm{MtCO}_{2} / \mathrm{yr}\right]$ & 3 & 10 & 30 & 3 & 10 & 30 \\
\hline IPCC & 5.75 & 2.95 & 1.75 & 8.05 & 3.85 & 2.15 \\
ZEP & 10.9 & 3.3 & 0 & 14.8 & 4.8 & \\
USDOE & 4.9 & & 1.7 & & & \\
\hline
\end{tabular}




\section{References}

Bauer, N., V. Bosetti, M. Hamdi-Cherif, A. Kitous, D. McCollum, A. Méjean, S. Rao, H. Turton, L. Paroussos, S. Ashina, K. Calvin, K. Wada, and D. van Vuuren (2015). CO2 emission mitigation and fossil fuel markets: Dynamic and international aspects of climate policies. Technological Forecasting and Social Change 90, 243-256.

Beér, J. M. (2007). High efficiency electric power generation: The environmental role. Progress in Energy and Combustion Science, 107-134.

Bielicki, J. M., C. A. Peters, J. P. Fitts, and E. J. Wilson (2015). An examination of geologic carbon sequestration policies in the context of leakage potential. International Journal of Greenhouse Gas Control 3\%, 61-75.

Catalanotti, E., K. J. Hughes, R. T. Porter, J. Price, and M. Pourkashanian (2014). Evaluation of performance and cost of combustion-based power plants with $\mathrm{CO}_{2}$ capture in the united kingdom. Environmental Progress and Sustainable Energy 33(4), 1425-1431.

Chum, H., A. Faaij, J. Moreira, G. Berndes, P. Dhamija, H. Dong, B. Gabrielle, A. Goss Eng, W. Lucht, M. Mapako, O. Masera Cerutti, T. McIntyre, T. Minowa, and K. Pingoud (2011). Bioenergy. in ipcc special report on renewable energy sources and climate change mitigation [o. edenhofer, r. pichs-madruga, y. sokona, k. seyboth, p. matschoss, s. kadner, t. zwickel, p. eickemeier, g. hansen, s. schlömer, c. von stechow (eds)].

Creutzig, F., E. Corbera, S. Bolwig, and C. Hunsberger (2013). Integrating place-specific livelihood and equity outcomes into global assessments of bioenergy deployment. Environmental Research Letters 8(3), 035047.

Davidson, C. L., R. T. Dahowski, H. C. McJeon, L. E. Clarke, G. C. Iyer, and M. Muratori (2017). The Value of CCS under Current Policy Scenarios: NDCs and Beyond. Energy Procedia 114, 7521-7527.

De Cian, E. and M. Tavoni (2012). Mitigation Portfolio and Policy Instruments When Hedging Against Climate Policy and Technology Uncertainty. Environmental Modeling $\&$ Assessment 17(1-2), 123-136.

DOE-NETL (2011). Cost and performance of PC and IGCC plants for a range of carbon dioxide capture. https://www . netl. doe.gov/File\%20Library/Research/Energy\% 20Analysis/Coal/Gerdes-08022011.pdf. 
Emmerling, J., L. Drouet, L. Reis, M. Bevione, L. Berger, V. Bosetti, S. Carrara, E. De Cian, G. de Maere d'Aertrycke, T. Longden, M. Malpede, G. Marangoni, F. Sferra, M. Tavoni, J. Witajewski-Baltvilks, and P. Havlík (2016). The WITCH 2016 Model - Documentation and Implementation of the Shared Socioeconomic Pathways. SSRN Scholarly Paper ID 2800970, Social Science Research Network, Rochester, NY.

GCCSI (2012). $\mathrm{CO}_{2}$ capture technologies: pre-combustion capture. http://www.globalccsinstitute.com/publications/ co2-capture-technologies-pre-combustion-capture.

GCCSI (2016). The global status of CCS 2016: summary report. http://status . globalccsinstitute. com/?v=2016.

GCCSI/EPRI (2012). $\quad \mathrm{CO}_{2}$ capture technologies: Oxy combustion with $\mathrm{CO}_{2}$ capture. https://www.globalccsinstitute.com/publications/ co2-capture-technologies-oxy-combustion-co2-capture.

Gibbins, J., H. Chalmers, M. Lucquiaud, J. Li, N. McGlashan, X. Liang, and J. Davison (2011). Techno-economic assessment of $\mathrm{CO}_{2}$ capture retrofit to existing power plants. Energy Procedia 4, 1835-1842.

Godec, M., V. Kuuskraa, T. Van Leeuwen, L. Stephen Melzer, and N. Wildgust (2011). $\mathrm{CO}_{2}$ storage in depleted oil fields: The worldwide potential for carbon dioxide enhanced oil recovery. Energy Procedia, 2162-2169.

Havlík, P., H. Valin, M. Herrero, M. Obersteiner, E. Schmid, M. C. Rufino, A. Mosnier, P. K. Thornton, H. Böttcher, R. T. Conant, S. Frank, S. Fritz, S. Fuss, F. Kraxner, and A. Notenbaert (2014). Climate change mitigation through livestock system transitions. Proceedings of the National Academy of Sciences 111(10), 3709-3714.

Heck, V., D. Gerten, W. Lucht, and A. Popp (2018). Biomass-based negative emissions difficult to reconcile with planetary boundaries. Nature Climate Change 8(2), 151-155.

IEA (2013a). Technology roadmap: Carbon capture and storage 2013. http://www.iea.org/publications/freepublications/publication/ technology-roadmap-carbon-capture-and-storage-2013.html.

IEA (2013b). Technology roadmap: Carbon capture and storage 2013. https://www. iea.org/publications/freepublications/publication/ technology-roadmap-carbon-capture-and-storage-2013.html. 
IEA (2015). Insight publications: Storing $\mathrm{CO}_{2}$ through enhanced oil recovery. https://www.iea.org/publications/insights/insightpublications/ storing-co2-through-enhanced-oil-recovery.html.

IEA (2016). Key world energy statistics 2016. http://dx.doi.org/10.1787/key_energ_ stat-2016-en.

IEAGHG (2009). Assessment of sub sea ecosystem impacts. https://www. globalccsinstitute.com/publications/assessment-sub-sea-ecosystem-impacts.

IEAGHG (2010). Oxyfuel combustion of pulverised coal. http://ieaghg.org/docs/ General_Docs/Reports/2010-07.pdf.

IEAGHG (2011a). Potential for biomass and carbon dioxide capture and storage. https: //www . eenews. net/assets/2011/08/04/document_cw_01.pdf.

IEAGHG (2011b). Retrofitting $\mathrm{CO}_{2}$ capture to existing power plants. https://www.globalccsinstitute.com/publications/ retrofitting-co2-capture-existing-power-plants.

IEAGHG (2012). $\mathrm{CO}_{2}$ capture at gas fired power plants. http://www. ieaghg.org/docs/ General_Docs/Reports/2012-08.pdf.

IEAGHG (2014a). Biomass and ccs - guidance for accounting for negative emissions. http: //www . ieaghg. org/docs/General_Docs/Reports/2014-05.pdf.

IEAGHG (2014b). $\mathrm{CO}_{2}$ capture at coal based power and hydrogen plants. http://ieaghg. org/docs/General_Docs/Reports/2014-03.pdf.

IPCC (2014). Climate Change 2014: Synthesis Report. Contribution of Working Groups I, II and III to the Fifth Assessment Report of the Intergovernmental Panel on Climate Change. https://www.ipcc.ch/report/ar5/syr/.

Jamasb, T. (2007). Technical Change Theory and Learning Curves: Patterns of Progress in Electricity Generation Technologies. The Energy Journal 28(3), 51-71.

Johnson, N., V. Krey, D. L. McCollum, S. Rao, K. Riahi, and J. Rogelj (2015). Stranded on a low-carbon planet: Implications of climate policy for the phase-out of coal-based power plants. Technological Forecasting and Social Change 90, 89-102.

Kaltschmitt, M., W. Streicher, and A. Wiese (Eds.) (2007). Renewable Energy: Technology, Economics and Environment. Berlin Heidelberg: Springer-Verlag. 
Karayannis, V., G. Charalampides, and E. Lakioti (2014). Socio-economic aspects of CCS technologies. Procedia Economics and Finance 14, 295-302.

Kemper, J. (2015). Biomass and carbon dioxide capture and storage: A review. International Journal of Greenhouse Gas Control 40.

Koelbl, B., M. van den Broek, B. van Ruijven, A. Faaij, and D. van Vuuren (2014). Uncertainty in the deployment of carbon capture and storage (CCS): A sensitivity analysis to technoeconomic parameter uncertainty. International Journal of Greenhouse Gas Control 27, 81-102.

Koelbl, B., M. van den Broek, B. van Ruijven, D. van Vuuren, and A. Faaij (2013). A sensitivity analysis of the global deployment of CCS to the cost of storage and storage capacity estimates. Energy Procedia 37, 7537-7544.

Koelbl, B. S. (2016). Deployment potential and macro-economic impacts of carbon dioxide capture and storage in the future energy system. Dissertation. Universiteit Utrecht. http: //dspace.library.uu.nl/handle/1874/339521.

Koelbl, B. S., M. A. v. d. Broek, A. P. C. Faaij, and D. P. v. Vuuren (2014). Uncertainty in carbon capture and storage (CCS) deployment projections: a cross-model comparison exercise. Climatic Change 123.

Krey, V., G. Luderer, L. Clarke, and E. Kriegler (2014). Getting from here to there - energy technology transformation pathways in the EMF27 scenarios. Climatic Change 123(3), 369-382.

Leeson, D., N. Mac Dowell, N. Shah, C. Petit, and P. S. Fennell (2017). A Techno-economic analysis and systematic review of carbon capture and storage (CCS) applied to the iron and steel, cement, oil refining and pulp and paper industries, as well as other high purity sources. International Journal of Greenhouse Gas Control 61, 71-84.

Leung, D. Y. C., G. Caramanna, and M. M. Maroto-Valer (2014). An overview of current status of carbon dioxide capture and storage technologies. Renewable and Sustainable Energy Reviews 39, 426-443.

Li, S., X. Zhang, L. Gao, and H. Jin (2012). Learning rates and future cost curves for fossil fuel energy systems with $\mathrm{CO}_{2}$ capture: Methodology and case studies. Applied Energy 93, 348-356. 
Lipponen, J., S. McCulloch, S. Keeling, T. Stanley, N. Berghout, and T. Berly (2017). The Politics of Large-scale CCS Deployment. Energy Procedia 114, 7581-7595.

Luderer, G., V. Bosetti, M. Jakob, M. Leimbach, J. C. Steckel, H. Waisman, and O. Edenhofer (2012). The economics of decarbonizing the energy system - results and insights from the RECIPE model intercomparison. Climatic Change 114(1), 9-37.

Luderer, G., R. C. Pietzcker, S. Carrara, H. S. de Boer, S. Fujimori, N. Johnson, S. Mima, and D. Arent (2017). Assessment of wind and solar power in global low-carbon energy scenarios: An introduction. Energy Economics 64, 542-551.

MacDonald, M. (2012). Potential cost reductions in CCS in the power sector. https: //ukccsrc.ac.uk/system/files/publications/ccs-reports/DECC_CCS_360.pdf.

Mander, S., K. Anderson, A. Larkin, C. Gough, and N. Vaughan (2017). The Role of Bio-energy with Carbon Capture and Storage in Meeting the Climate Mitigation Challenge: A Whole System Perspective. Energy Procedia 114, 6036-6043.

McCoy, S. T. and E. S. Rubin (2008). An engineering-economic model of pipeline transport of $\mathrm{CO}_{2}$ with application to carbon capture and storage. International Journal of Greenhouse Gas Control 2(2), 219-229.

Meinshausen, M., S. C. B. Raper, and T. M. L. Wigley (2011). Emulating coupled atmosphereocean and carbon cycle models with a simpler model, MAGICC6 - part 1: Model description and calibration. Atmos. Chem. Phys. 11(4).

Metz, B., O. Davidson, H. de Coninck, M. Loos, and L. Meyer (2005). IPCC Special Report on Carbon Dioxide Capture and Storage. Prepared by Working Group III of the Intergovernmental Panel on Climate Change.

Muratori, M., K. Calvin, M. Wise, P. Kyle, and J. Edmonds (2016). Global economic consequences of deploying bioenergy with carbon capture and storage (BECCS). Environmental Research Letters 11(9), 095004.

Muratori, M., H. Kheshgi, B. Mignone, L. Clarke, H. McJeon, and J. Edmonds (2017). Carbon capture and storage across fuels and sectors in energy system transformation pathways. International Journal of Greenhouse Gas Control 57, 34-41.

Neij, L. (2008). Cost development of future technologies for power generation-A study based on experience curves and complementary bottom-up assessments. Energy Policy 36(6), 2200-2211. 
OECD/IEA (2010). Cost and performance of carbon dioxide capture from power generation. https://www.iea.org/publications/freepublications/publication/ costperf_ccs_powergen.pdf.

Rogelj, J., G. Luderer, R. C. Pietzcker, E. Kriegler, M. Schaeffer, V. Krey, and K. Riahi (2015). Energy system transformations for limiting end-of-century warming to below 1.5 ${ }^{\circ} \mathrm{C}$. Nature Climate Change 5(6), 519-527.

Rubin, E. S., I. M. Azevedo, P. Jaramillo, and S. Yeh (2015). A review of learning rates for electricity supply technologies. Energy Policy 86, 198-218.

Rubin, E. S., J. E. Davison, and H. J. Herzog (2015). The cost of CO2 capture and storage. International Journal of Greenhouse Gas Control 40, 378-400.

Rubin, E. S., S. Yeh, M. Antes, M. Berkenpas, and J. Davison (2007). Use of experience curves to estimate the future cost of power plants with $\mathrm{CO}_{2}$ capture. International Journal of Greenhouse Gas Control 1(2), 188-197.

Van den Broek, M., P. Veenendaal, P. Koutstaal, W. Turkenburg, and A. Faaij (2011). Impact of international climate policies on $\mathrm{CO}_{2}$ capture and storage deployment: Illustrated in the dutch energy system. Energy Policy 39, 2000-2019.

van der Zwaan, B. and K. Smekens (2009). $\mathrm{co}_{2}$ capture and storage with leakage in an energy-climate model. Environmental Modeling \&3 Assessment 14(2), 135-148.

Van der Zwaan, B. and M. Tavoni (2011). Coal-based power generation with CCS versus nuclear energy: Two competitive base-load climate control options. Energy Procedia 4 , $5830-5837$.

Van Der Zwaan, B. C. C., H. Rösler, T. Kober, T. Aboumahboub, K. V. Calvin, D. E. H. J. Gernaat, G. Marangoni, and D. McCollum (2013). A cross-model comparison of global long-term technology diffusion under a $2^{\circ} \mathrm{C}$ climate change control target. Climate Change Economics 04 (04), 1340013.

Vinca, A., J. Emmerling, and M. Tavoni (2018). Bearing the Cost of Stored Carbon Leakage. Frontiers in Energy Research 6.

Vuuren, D. P. v., A. Hof, D. Gernaat, and H. S. de Boer (2017). Limiting global temperature change to $1.5{ }^{\circ} \mathrm{C}$ - PBL Netherlands Environmental Assessment Agency Report. http://www.pbl.nl/en/publications/ limiting-global-temperature-change-to-1-5-degree-celsius. 
Whittaker, S. and A. Kneppers (2013). Lessons in the development of large-scale $\mathrm{CO}_{2}$ storage projects. Energy Procedia 37, 3646-3654.

Wu, X. D., Q. Yang, G. Q. Chen, T. Hayat, and A. Alsaedi (2016). Progress and prospect of CCS in China: Using learning curve to assess the cost-viability of a $2 \times 600 \mathrm{MW}$ retrofitted oxyfuel power plant as a case study. Renewable and Sustainable Energy Reviews 60, $1274-1285$.

ZEP (2011a). The costs of $\mathrm{CO}_{2}$ storage. http://www .zeroemissionsplatform.eu/library/ publication/168-zep-cost-report-storage.html.

ZEP (2011b). The costs of $\mathrm{CO}_{2}$ transport. http://www.globalccsinstitute. com/sites/www.globalccsinstitute.com/files/publications/17011/ costs-co2-capture-transport-and-storage.pdf.

ZEP/EBTP (2012). Biomass with $\mathrm{CO}_{2}$ capture and storage (Bio-CCS) - the way forward for Europe. European Technology Platform for Zero Emission Fossil Fuel Power Plants. http: //www .etipbioenergy.eu/images/EBTP-ZEP-Report-Bio-CCS-The-Way-Forward.pdf.

\section{List of Figures}

1 Electricity generation mix by CCS plants over time, for the 2DC (upper panel) and the 1.5DC (lower panel) scenarios . . . . . . . . . . . . . 13

2 Percentage variation in cumulative installed CCS capacity in response to changes in six performance parameters . . . . . . . . . . . . . . 15

3 Cumulative electricity generation from CCS plants by 2100 for different temperature increase targets . . . . . . . . . . . . . . . . . . 17

4 Variation in installed CCS capacity over temperature increase, in response to changes in efficiencies and investment cost . . . . . . . . . . . . . . . 18

5 Comparison of CCS and intermittent RES share of yearly total electricity generation, across different climate targets. . . . . . . . . . . . . .

6 Average temperature increase for 2DC and 1.5DC scenarios with two confidence interval areas. The dark shading denotes the $25 \% / 75 \%$ percentile region, and the light shading the $17 \% / 83 \%$ percentile region. The lines in the middle represent the median. . . . . . . . . . . . . . . . . . . . . . 21

$7 \quad$ Electricity generation mix for $1.5 \mathrm{DC}$ and $2 \mathrm{DC}$ scenarios. . . . . . . . . . 22 


\section{List of Tables}

1 Main performance parameters of power plants with CCS as results of the literature review. . . . . . . . . . . . . . . . . 8

2 Investments and O\&M cost of Power plants with and w/o CCS as results of the literature review $(2005$ USD) . . . . . . . . . . . . . . 9

3 Renewable energy technologies input data. These are representative of the Western Europe region, values differ regionally. Sources (Neij, 2008; Kaltschmitt et al., 2007) . . . . . . . . . . . . . . . . . . . 22

$4 \quad \mathrm{CO}_{2}$ storage capacity divided per region in low, best, high cases in $\left[\mathrm{GtCO}_{2}\right]$ (IEAGHG, 2011a). . . . . . . . . . . . . . . . . . . 23

5 Percentage of onshore and offshore capacity for storage potential of O\&G and Aquifers (IEAGHG, 2009). . . . . . . . . . . . . . . 23

6 Cost of storage for O\&G sources and Saline formations $\left[\$ / \mathrm{tCO}_{2}\right](\mathrm{ZEP}, 2011 \mathrm{a}) .23$

7 Transport costs on a common basis for onshore and offshore pipelines at three different capacities $\left[\$ / \mathrm{tCO}_{2} / 250 \mathrm{~km}\right]$ (Rubin et al., 2015). Original sources are reported in the first column: the values have been converted in the same unit of measure by the author to ease the comparison. . . . . . . . . . . . . . . 24

8 Data gathered during our literature review. . . . . . . . . . . . . 25 